\title{
A Goniophotometric Study of the Iridescent Effect in Tamamushi Fabrics
}

\author{
By Tsuneyo Tsuboi*, Koji Nihira* and Toshihiro Gunji**, Members, TMSJ \\ *Faculty of Engineering, Meiji University, Kanagawa-ken \\ **Nitto Boseki Co. Ltd., Osaka \\ Based on the Journal of the Textile Machinery Society of Japan, Transactions, Vol. 26, No. 6, T103-112(1973)
}

\begin{abstract}
This paper discusses the optical properties of the so-called Tamamus. i Fabrics (iridescent fabrics) measured with a goniophotometer.

The results obtained are:

(1) The factors governing the optical properties of Tamamushi fabrics are considered to be the surface structure of the fabrics, fabric construction, gloss and combination of the colors of warp ends and filling yarns.

(2) In order to analyse their optical properties, the reflected light from Tamamushi fabrics should be separated into the specular and the diffuse components.

(3) The optical properties of warp ends and filling yarns have an important bearing on the iridescence of Tamamushi fabrics.

(4) The color change of Tamamushi fabrics should be discussed from the goniophotometric reflection curves obtained under a monochromatic light resembling the warp ends and filling yarns in color.

(5) The larger the hue difference between the warp ends and filling yarns, the larger the change of color on the chromatic diagram.

\section{KEY WORDS: Reflectometer, Appearance, Surface properties, Woven fabrics, Fabric Design, Reflectance, Color, Color Designation, Colorimeters, Color Measurement, Gloss, Luster}

\section{Introduction}

Tamamushi fabric (iridescent fabric) is densely woven with different colored yarns in warp and filling. Different colors show up when the fabric is viewed at different angles or in different directions. Although Tamamushi fabric has been made of silk, now a variety of synthetic fibers are woven into it, and used for cloth linings, dress materials, etc.

There have been very few works published which dealt with Tamamushi fabric, inspite of its long history, except Tanaka's ${ }^{[1]}$ and Sawaji's ${ }^{[2]}$ papers.

Tanaka studied the iridescent effect of Tamamushi fabric and obtained some spectral reflection curves with a Beckman type photo-electric spectrophotometer. In his paper he concludes that (1) the iridescent effect is a kind of color mixing produced by the reflection difference between warp and filling, and (2) wide color change needs large color difference between warp and filling.

It is tried in this paper to obtain a reflection distribution curve in each direction of warp, filling and $45^{\circ}$, by using a goniophotometer, and further to examine the spectral reflection properties by using a goniophotometer equipped with an interference filter. Then are discussed (1) the mechanism of the reflection from Tamamushi fabric sur- face, (2) the correlation between the uneven distribution of infinitely small surfaces on the fabric ${ }^{[3]}$ and the appearance of the iridescent effect, and (3) goniophotometric color measurement.

\section{Theory}

The iridescent effect of Tamamushi fabric is produced by the three-dimensional pattern of the fabric surface. The nature of the pattern is decided by various factors related with fibers, yarns and fabrics (e.g., staple or filament, fiber length, fiber material, yarn twist, fabriz density and structure, etc.).

Tamamushi effect should be studied not only in terms of geometric structure but also in terms of the optical difference between warp and filling. Therefore, the difference between warp and filling in the gonio-photometric reflection distribution curve was measured using the goniophotometer, and the causes of the difference in optical properties were discussed.

The optical properties of single fibers and those of heavy multi-filaments obtained in the previous paper ${ }^{[4]}$ were used in this paper to discuss Tamamushi effect.

The change in view angle to the warp or filling of Tamamushi fabric influences the ratio of the color mixing at the 
receiving angle. It is an important problem for Tamamushi effect how the reflection lights from the different colored warp or filling varies with the view angle. So it is desired that the reflected lights from different colored warp and filling illuminated with a light source $A$ can be separated from each other and measured for each of them (warp and filling). However, since it is very difficult, the goniophotometric curves were recorded by using interference filters resembling the warp and the filling in color. From these results, the goniophotometric reflection curves for the warp and the filling were inferred. Then the validity of the inference referring to the reflection distribution curve for the heavy multifilaments was discussed.

From recorded reflection curves in warp and filling directions of Tamamushi fabric, the relation between the warp and the incident luminous flux and that between the filling and theincidence luminous flux were arranged under several conditions of measurement as follows:

1) Measurement of reflection distribution in warp direction

In this case the photometric plane is arranged parallel with the warp and yet perpendicular to the filling.

2) Measurement of reflection distribution in filling direction In this case the photometric plane is arranged vertical to the filling and yet parallel with the warp.

The results thus obtained can be used as the reference data.

The heavy multi-filament has almost no twisting. The single fibers are arranged in parallel and linear state. However in actual fabrics, yarns are a little twisted and zigzag due to weaving. This is important for determining the pattern of the goniophotometric curves for warps and fillings. Three-dimensional pattern has an important bearing on the pattern of the reflected light distribution curves for warps and fillings.

The reflected light from the fabric surface is separated into a specular and a diffuse reflection light.

The specular reflection light is considered to have the light source color and the diffuse reflection light from the objectcolor. Therefore, the diffuse reflection light is thought to have an important bearing on the iridescent effect.

The specular reflection gives gloss and adds a hue to the object color, and leads the latter to have the color similar to the light source color. Besides, it determines the quality of the gloss on the fabric surface. The reflection properties were discussed by taking these points into consideration on the assumption that each of the incident luminous flux and the receiving luminous flux is parallel in itself.

\section{2-1. Specular reflection}

The specular reflection light is obtained from the reflection perpendicular to the photometric plane.

The specular reflection $\Delta I s p$ from the infinitely small

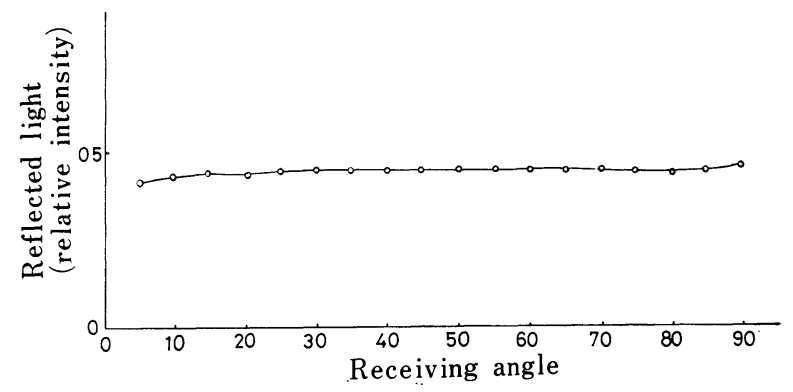

Fig. 1 Goniophotometric reflection curve of the right column (parallel luminous flux density: 1)

surface $A s$ perpendicular to the photometric plane is

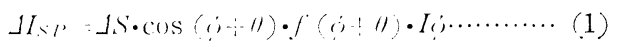

were $\phi:$ incident angle, $\theta$ : inclination angle of $\Delta s, I \phi$ : incident luminous flux per unit area and $f(\phi+\theta)$ : Fresnel's coefficient.

$\Delta i s p$ is the specular reflection light in the direction of $\dot{\phi}=\dot{\phi}+2 \theta$.

Herce,

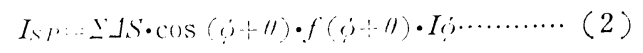

Fig. 1 shows the result calculated from the goniophotometric reflection curves for the incidence perpendicular to the axis of the monofilament. The goniophotometric reflection curves of the transparent body (reflective index: 1.5) take a flat form. Those for filament are different from that shown in Fig. 1, because actual filaments are not straight and ordinarily deformed.

\section{2-2. Diffuse reflection light}

The diffuse reflection light cannot be dealt with twodimensionally, as it is different from the specular reflection light. When the parallel luminous flux was reflected at the incident angle $\phi$ against the plane of the fabric, and the

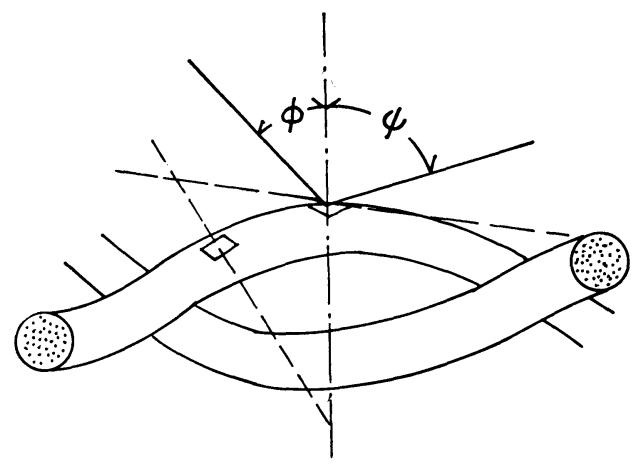

(a)

Fig 2 (a) Ircident light and reflected light on the fabric surface 


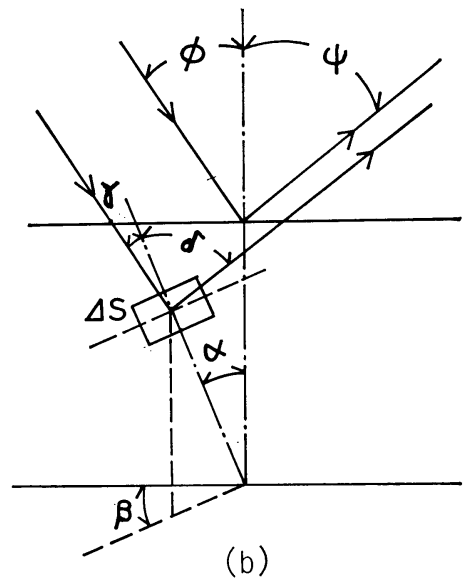

(b)

Fig. 2 (b) Incident light and reflected light of the fabric surface

angle between the normal of the infinitely small surface $\Delta S$ (in Fig. 2), and the incident axis $\phi$ is $\gamma$, then the incident luminous flux is reflected at the incident angle $\gamma$ against $\Delta S$. Hence, if the incident light per unit area is $I \phi$, the incident luminous flux against $\Delta S$ is

$$
\Delta I_{r}=\Delta S \cdot \cos r \cdot I_{j} \cdot
$$

Assuming the diffuse reflection function of the fabric surface to be $H$, it is the function of both the incident angle and the reflection angle. As shown in Fig. 2, if the angle between the reflection light in the direction $\psi$ and the normal line of $\Delta S$ is $\delta$, then the incident angle and the reflection angle against $\Delta S$ are $\gamma$ and $\delta$, respectively. Hence, the diffuse reflection light $\Delta I_{d f}$ by $\Delta S$ in the direction $\psi$ is

$$
\Delta I_{l t} f=\Delta S \cdot \cos r \cdot I t \cdot H(r ; \quad j)
$$

However, from Fig. 3,

$$
\begin{aligned}
& r=\cos ^{-1}(\cos x \cdot \cos y \cdot \sin x \cdot \sin \theta \cos 3 \cdots(5) \\
& j=\cos ^{-1}(\cos \alpha \cdot \cos \xi-\sin \alpha \sin \varphi \cos ; \cdots(6)
\end{aligned}
$$

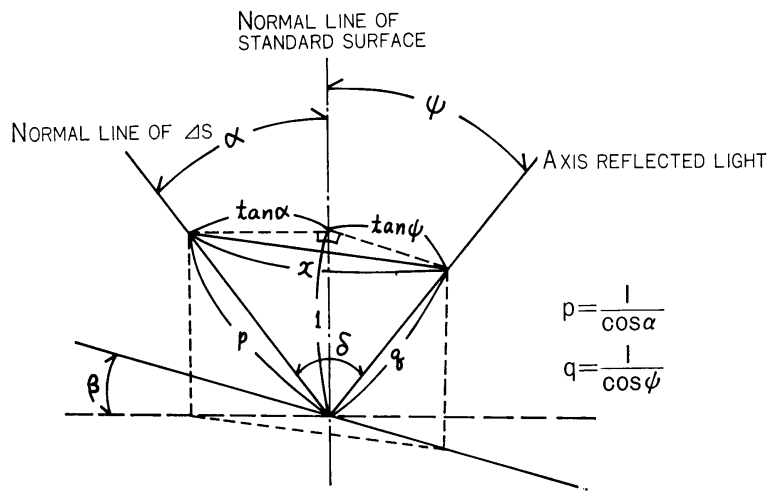

Fig. 3 Relation among $\alpha, \phi$ and $\delta$.
The diffuse reflection light at the receiving angle $\phi$ is the collection of the shaded parts behind $\Delta S$, i.e.,

$$
I_{d j}(\dot{\varphi})=\Sigma d \cdot \cos r \cdot I \dot{I} \cdot H(r, \quad \delta
$$

Substituting $\gamma$ and $\delta$ from eqs. (5) and (6) into eq. (7), $I_{d f}$ is obtained as the function of such angles as the incident angle $\phi$, the reflection angle $\phi$, and the inclinations $\alpha$ and $\beta$ of $\Delta S$.

If the diffuse reflection function $H(\gamma, \delta)$ is of the perfect diffusing surface, it will show a spherical surface according to Lambert's law. But it is not proved for general samples.

In this study, however, with such rugged surface as Tamamushi fabric, the specular reflection is evenly distributed, and the light quantity due to it may be thought small. Since reflection lights are difficult to separate, the problem was discussed by using the goniophotometric reflection curve. It may be very difficult, however, to calculate with more complex fabric surface.

2-3. Goniophotometric reflection curve of yarns

Figs. 4 and 5 show the goniophotometric reflection

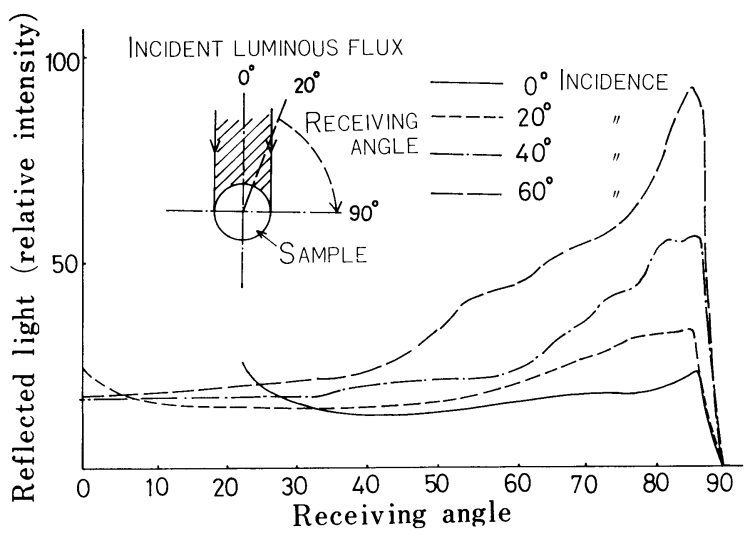

Fig. 4 Goniophotometric reflection curve of the circular cross-section fiber (polypro-heavy multi-filament) I

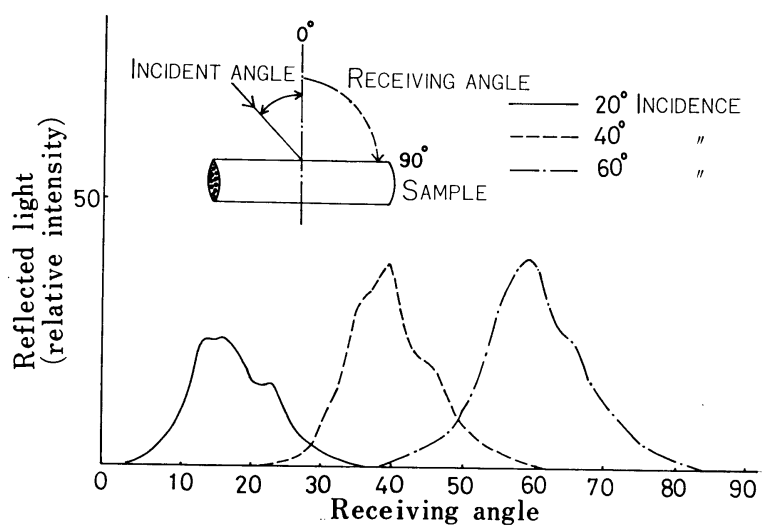

Fig 5 Goniophotometric reflection curve of the circular cross-section fibers (polypro-heavy-multi-filament) II 
curves of the twistless mono-filament. Fig. 4 shows the case where the photometric plane is perpendicular to the yarn axis, Fig. 5 the case where it is parallel with the yarn axis.

As is clear in Figs. 4 and 5, the maximum value of the reflected light in the goniophotometric reflection curve in the direction perpendicular to the axis exists at around the receiving angle of $90^{\circ}$. The larger the incident angle the larger the quantity of the reflected light. This tendency was acknowledged in the goniophotometric reflection curve of Tamamushi fabric. A goniophotometric reflection curve observed in the direction parallel with the axis is represented by a curve having its maximum value in the position of the receiving angle equal to the incident angle. If the straight yarn is bent, the increase of the incident angle shifts the position of the maximum value of the reflected light slightly toward the increasing direction of the receiving angle. That is, the reflected light will have the maximum value at around $90^{\circ}$ on the surface with an incident light of $45^{\circ}$ at an inclination angle of $22.5^{\circ}$. Accordingly, if the yarn is bent by weaving, the reflection distribution curve shows the maximum value at around the receiving angle of $90^{\circ}$. In other words, when the yarn is straight, the maximum value appears at the receiving angle tantamount to the incident angle. As the bending increases, the angle of the receiving angle showing the maximum value reaches $90^{\circ}$. The tendency of the reflection distribution from the yarn surface was clear from this experiment. The reflected light is the sum of the specular and the diffuse reflection lights. As the specular reflection light is evenly distributed on the uneven surface of the fabric, it is unnecessary to discuss the specular reflection light separately, and the summation of the specular and the diffuse reflection lights may be used to clarify the problem.

The tendency of the goniophotometric reflection curve varies by the direction whether or not the warp (or filling) is perpendicular to (or parallel with) the photometry plane. When the reflected light quantity from the warp or filling varies at a certain receiving angle, the ratio of the color

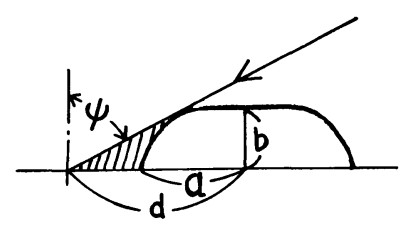

CROSS-SECTIONAL VIEW

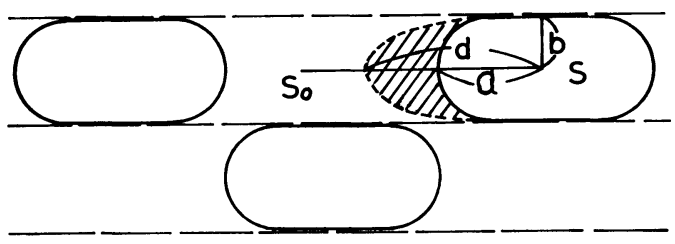

Fig. 6 mixing due to warps and fillings at the receiving angle varies.

As illustrated in Fig. 6, the convex part of the texture may be considered an ellipsoid and the valley part a flat plane, and they are assumed arranged uniformly on the fabric surface. The shaded part in the figure caused by the parallel luminous flux on to this surface can be obtained as follows:

If the shaded length in the central portion of the ellipsoid due to the parallel luminous flux at the receiving angle $\psi$ is $d$, then

$\mathrm{d}=\sqrt{a^{2}+b^{2} \tan ^{2} \phi}$

Hence, the shaded part is regarded as an ellipsoid enclosed with minor axis b and major axis d. So, the shaded area $s$ is

$$
S=\frac{\pi d b-\pi a b}{2}
$$

When $\phi=C$ (that is, in the normal direction), the area ratio of the conves to the valley is

$$
S: S_{0}: \cdots,-\pi b: S_{0}
$$

Then, the ratio $S / \varsigma_{0}$, that is the observed convex area divided by the valle; r rea when $\phi \neq 0$ on the receiving side is

$$
S: S_{0}:=\frac{\pi b(d+a)}{2}: S_{0} \cdots \frac{\pi b(d-a)}{2} \cdots(9)
$$

Fig. 7 shows calculated curves of these $S, S_{0}$ and $S_{0} / S$ when the major axis $a=2$ and the minor axis $b=1$. It is considered that, when $a=2$ and $b=1$, the surface is comparatively uneven. However, the ratio of $a$ to $b$ does not change until $45^{\circ}$. Beyond that limit, there arises a change depending upon the observed direction.

As is explained above, the iridescent effect of Tamamushi fabric is produced

1) by difference, in goniophotometric reflection curves,

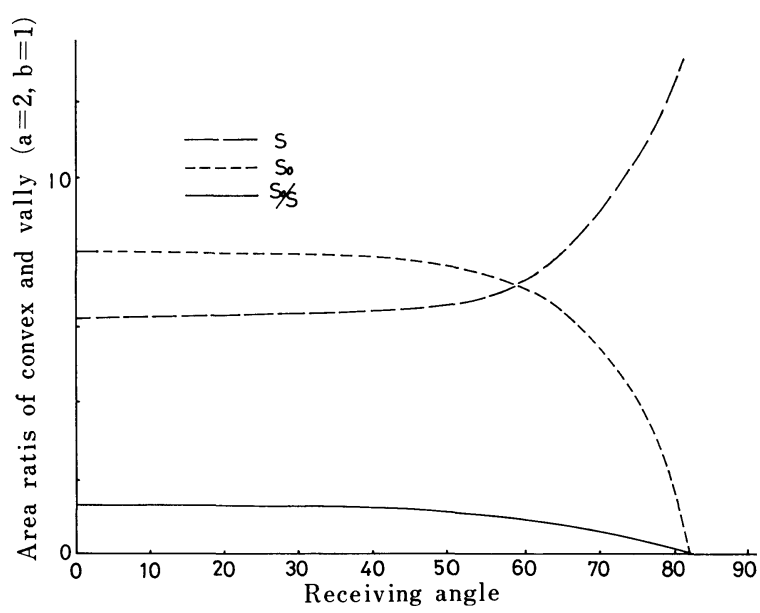

Fig. 7 Relation between the inciderce angla and $S_{0} / S$ 
arising from the difference in the reflection properties between warps and fillings. In other words, by the change in the ratio of lights reflected from warps and fillings,

2 ) by the ratio of the specular or the diffuse reflection lights, and

3) by the change in the ratio of the convex to the valley areas when the reflected angle $\phi$ increases.

\section{Method of Experiment}

\section{3-1. Experimental equipment}

The goniophotometer used in this experiment is illustrated in Fig. 8. The light source and the collimator are fixed and the telescope can be revolved without touching the collimator. One set of sample holders can be revolved freely. The plane sample $(50 \mathrm{~mm} \times 50 \mathrm{~mm})$ is set on the sample holders.

Outline of the goniophotometer is as follows:

Light source: Tangsten filament lamp (20V, 5A)

Collimator lens: $\mathrm{f}=265 \mathrm{~mm}$

Aperture of parallel luminous flux: within $1^{\circ}$
Diameter of parallel luminous flux : $20 \mathrm{~mm}$

Telescope lens: $\mathrm{f}=67 \mathrm{~mm}$

Aperture of receiving luminous flux: within $1^{\circ}$

Photo-multiplier tube: RCA-1P21

\section{3-2. Sample}

The sample used in this experiment is acetate twill. Red, yellow, green or blue yarns are used in warps and fillings as shown in Table 1. Photographs of their surfaces are shown in Fig. 9, their cross sections in Fig. 10.

\section{3-3. Experimental method}

1) Measurement by goniophotometer ${ }^{[5]}$

Goniophotometric reflection curves in warp, filling and

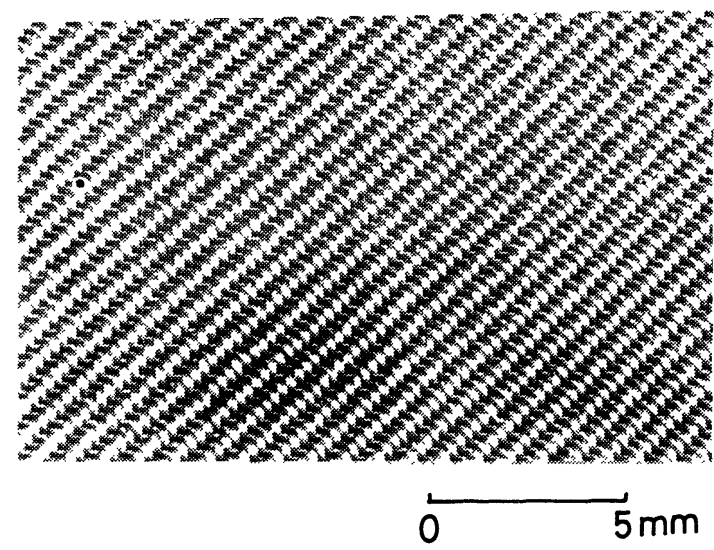

Fig. 9 Surface of Tamamushi fabric (Sample No. 1)

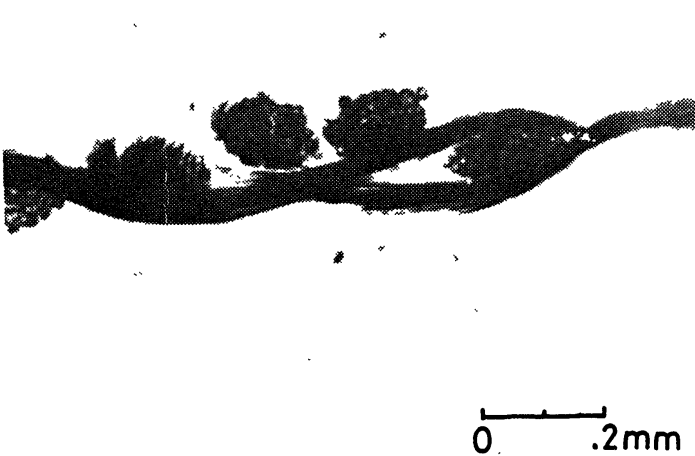

Fig. 10 Cross-section of Tamamushi fabrio (Sample No. 3)

Fig 8 Schematic diagram of goniophotometer

Table 1 Fabric Samples

\begin{tabular}{|c|c|c|c|c|c|}
\hline No. & Material & Yarn used & Structure & $\begin{array}{l}\text { Density } \\
\text { (per square inch) }\end{array}$ & Color \\
\hline 1 & Acetate-twill & $\begin{array}{r}75 \mathrm{~d} \text { Bright } \times \\
75 \mathrm{~d} \text { Bright }\end{array}$ & $2 \times 2$ Twill & 204 warps $\times 110$ fillings & $\begin{array}{l}\text { Warp: Biue } \\
\text { Filling: Red }\end{array}$ \\
\hline 2 & Acetate-twill & $\begin{array}{r}\text { 75d Bright } \times \\
\text { 75d Bright }\end{array}$ & $1 \times 3$ Twill & 172 warps $\times 112$ fillings & $\begin{array}{l}\text { Warp: Green } \\
\text { Filling: Yellow }\end{array}$ \\
\hline 3 & Acetate-twill & 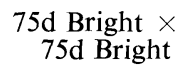 & $1 \times 3$ Twill & 152 warps $\times 116$ fillings & $\begin{array}{l}\text { Warp: Yellow } \\
\text { Filling: Re } 1\end{array}$ \\
\hline
\end{tabular}


$45^{\circ}$ directions were continuously recorded from $0^{\circ}$ to $90^{\circ}$ at incident angles $45^{\circ}$ and $60^{\circ}$. The same procedure was taken next with a goniophotometer equipped with interference filters having similar hue to that of the warp or filling.

2) Goniophotometric color measurement ${ }^{[6]}$

Color measurement was made with a goniophotometer equipped with interference fllters. Method of the color measurement for CIE system-tristimulus values, $X, Y$ and $Z$, was used here. Thirteen interference filters were used at the same intervals from $400 \mathrm{~nm}$ to $700 \mathrm{~nm}$. The ratio of the reflected light quantity from the standard white surface to that from the sample was measured at the given incidence and receiving angles. Tristimulus values, $X, Y$ and $Z$, and the derived $\mathrm{x}$ and $\mathrm{y}$ on the chromatic diagram were calculated as usual.

The tristimulus values $X, Y$ and $Z$ are given by

$$
\begin{aligned}
& X=k \sum_{\lambda=400}^{700} P_{\lambda} \cdot \overline{x_{\lambda}} \cdot \rho_{\lambda} \\
& Y=k \sum_{\lambda=400}^{700} P_{\lambda} \cdot \overline{y_{\lambda}} \cdot \rho_{\lambda} \\
& Z=k \sum_{\lambda=400}^{700} P_{\lambda} \cdot \overline{z_{\lambda}} \cdot \rho_{\lambda}
\end{aligned}
$$

where $P_{\lambda} \cdot$ the amount of radiation in watts between the wavelength limits $\lambda$

$\overline{x_{\lambda}}, \overline{y_{\lambda}}, \overline{z_{\lambda}}$ : spectral tristimulus values

$\rho_{\lambda}:$ the spectral luminous factor

$k: k=1 / \sum_{\lambda=400}^{700} P_{\lambda} \cdot \overline{y_{\lambda}}=1 / 100000$

The unit co-ordinates $x$ and $y$ will be given by

$$
\begin{aligned}
& x=X /(X+Y+Z) \\
& y=Y /(X+Y+Z)
\end{aligned}
$$

\section{Experimental Results and Discussion}

4-1. Results measured by goniophotometer

Goniophotometric reflection curves in warp and filling directions of sample No. 1 at incident angles of $45^{\circ}$ and $60^{\circ}$ are shown in Fig. 11. There is a difference between the curves depending upon the directions in which the measurement was done, namely in warp or in filling direction, but the goniophotometric reflection curve is the sum of lights reflected from both warp and filling.

By using monochromatic light source having the similar hue to that of the warp or filling, the goniophotometric reflection curve from each of warp and filling was recorded and is shown in Fig. 12.

When the fabric is measured in the filling direction, the cross-section of the fabric is shown in Fig. 13. When the interference filter having the similar hue to that of the filling yarn is used, the reflection from the warp having the quite different hue from that of the filling decreases largely. Hence, it is considered that the reflection from the filling has solely a great influence on the light quantity measured

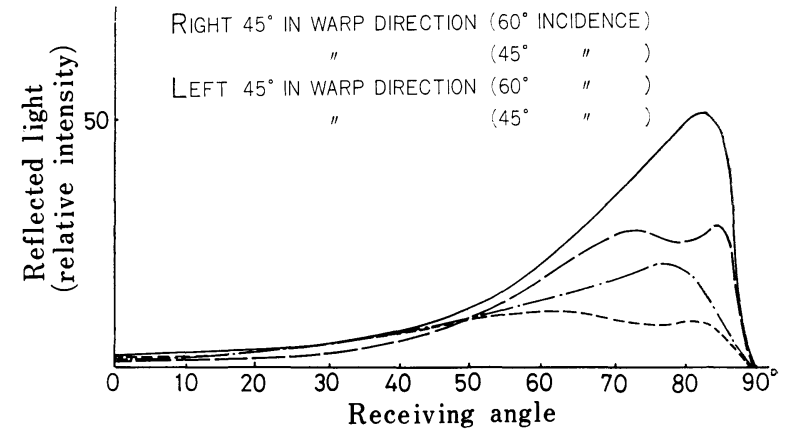

Fig. 11 Coniopl otomzric reflertion curves of Taramushi fatr'cs (Sampie No. !)
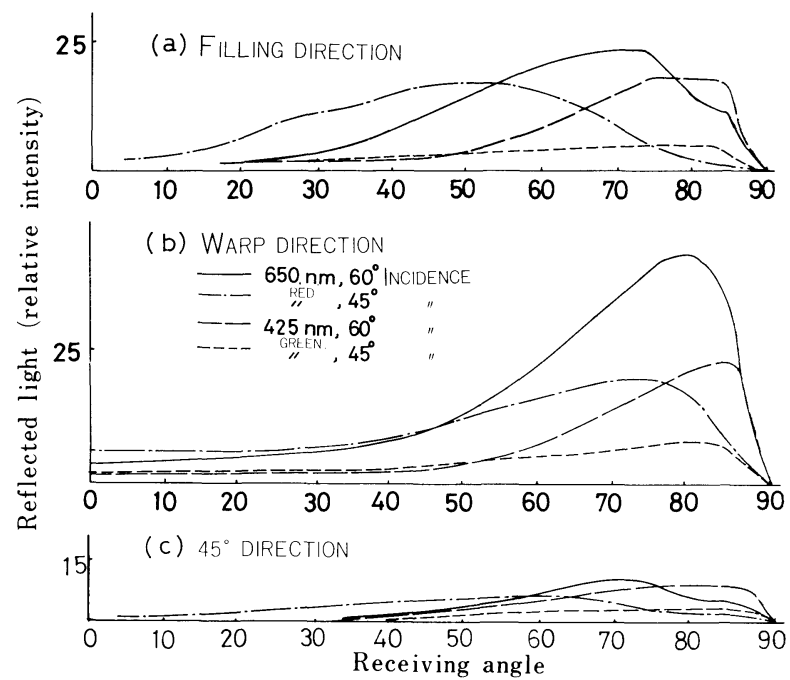

Fig. 12 Goniophotomstric refectim surves of Tamanushi fabrics (Sample k̃o. 1)

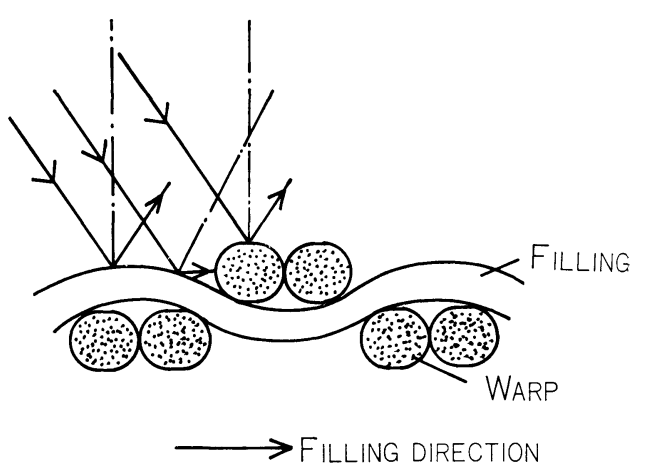

Fig. 13 Model of fabric cross-section

in the filling direction. Since the incident light is paralleI with the filling yarns, there is produced the diffuse reflection in the center of the specular reflection direction. In the case of fabric, however, the maximum value does not always arise at the regular reflected angle, but rather at the larger receiving angle. This is caused by the unevenness of the fabric surface. When the receiving angle increases, the 
reflected light from the warp decreases because it is shaded by the filling.

When using the interference filter having the similar hue to that of the warp, the warp gives a great influence to the reflected light. When measured in the filling direction, the incident light is perpendicular to the warp, and the reflection curve has the similar tendency to that of the reflection for the incidence perpendicular to the yarn, that is, the maximum value at around $90^{\circ}$.

When the reflected light is measured in the warp direction, the relation between the warp and the filling is inverse to that measured in the filling direction. When the interference filter having the similar hue to that of the filling is used, the maximum value arises at a large receiving angle. When the interference filter having the similar bue to that of the warp is used, the reflected light is influenced by the warp.

When the reflected light is measured at the angles $45^{\circ}$ left and $45^{\circ}$ right direction against the warp, the influence of the twill line of the fabric surface is markedly noticed, and the influence in the warp and the filling directions are also recognized. The results thus obtained are shown in Fig. 14. As the warp is floating while the filling is sinking, it is recognized that the reflection from the filling partially decreases due to the filling hiding behind the warp at over $75^{\circ}$, when the interference filter resembling the filling in color is used.

The goniophotometric reflection curve for the sample illuminated with a light source $A$ is the sum of those curves previously obtained with the warp and the filling by using filters resembling the warp and the filling in hue. Therefore, the tendency of goniophotometric reflection curve with the warp or the filling can be deduced by using two interference filters. In other words, it is understood that the color mixing can be made by summing up respective reflection curves for the warp and the filling.

The experimental results of sample No. 2 is shown in Fig. 15. The tendency in the goniophotometric curve does not vary by changing the incidence wave length when the difference between warp color and filling color is small.

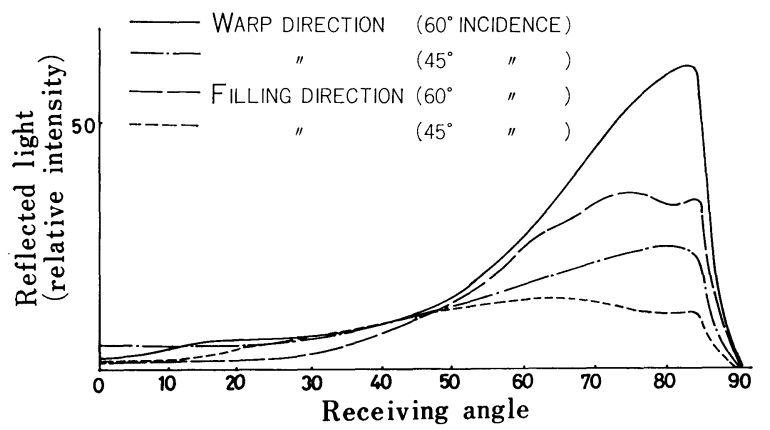

Fig. 14 Goxiophotometric raflaction curves of Tamamushi fabrics

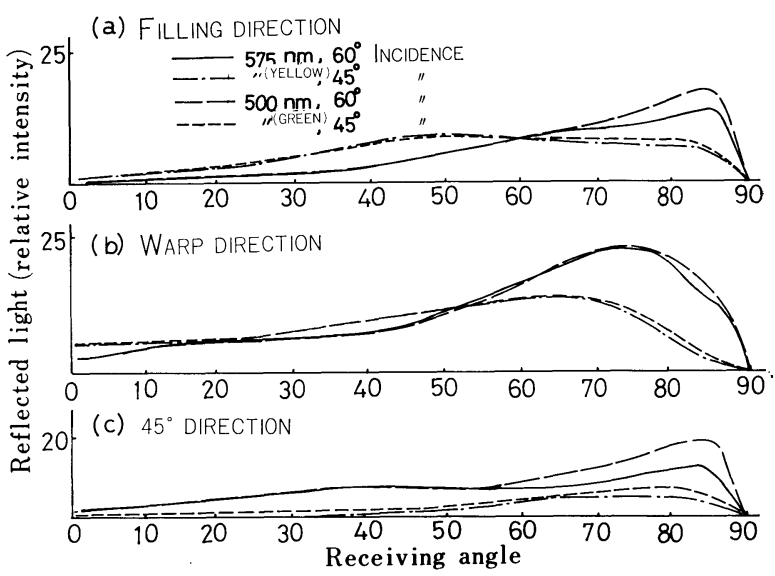

Fig. 15 Gonioohotometric reflection curves of Tamamushi fabrics (Sample No. 2)

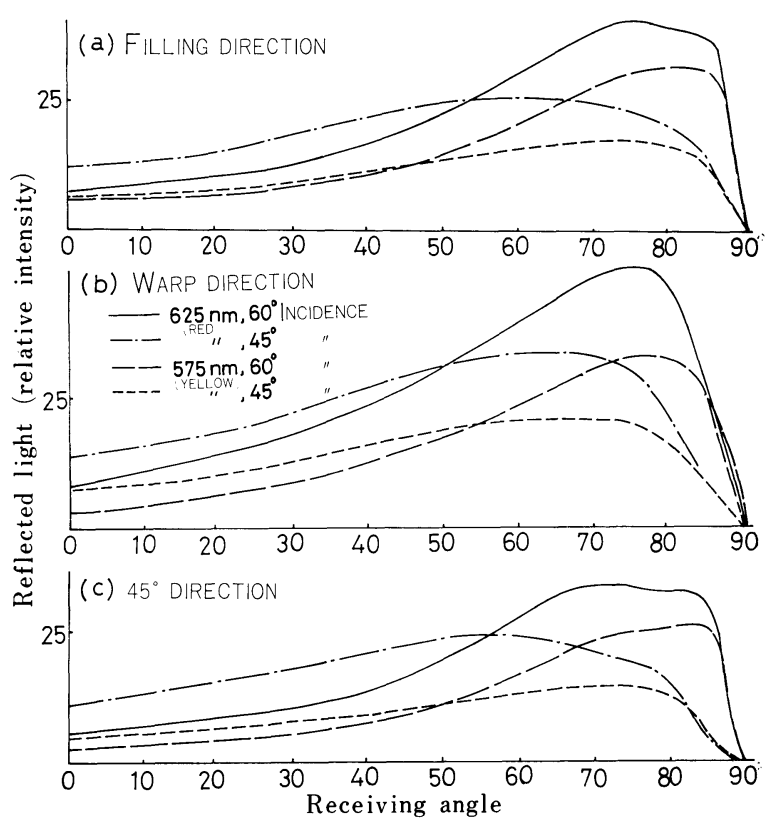

Fig. 16 Goniophotometric reflection curves of Tamamushi fabrics (Sample No. 3)

The measured results for sample No. 3 (Fig. 16) shows the same tendency as with sample No. 2, presumably because of small hue difference. However, with this sample, the difference between the reflected light quantity from the warp and that from the filling may be caused because of the brightness difference between the warp and the filling.

\section{4-2. Goniophotometric color measurement}

When the incidence angle is $45^{\circ}$, comparison was made, with sample No. 1 , between the standard white surface and the sample at the receiving angles of $0^{\circ}, 45^{\circ}, 60^{\circ}, 70^{\circ}$ and $80^{\circ}$ by using a monochromatic light through thirteen interference filters (Fig. 17). This curve is considered resembling 


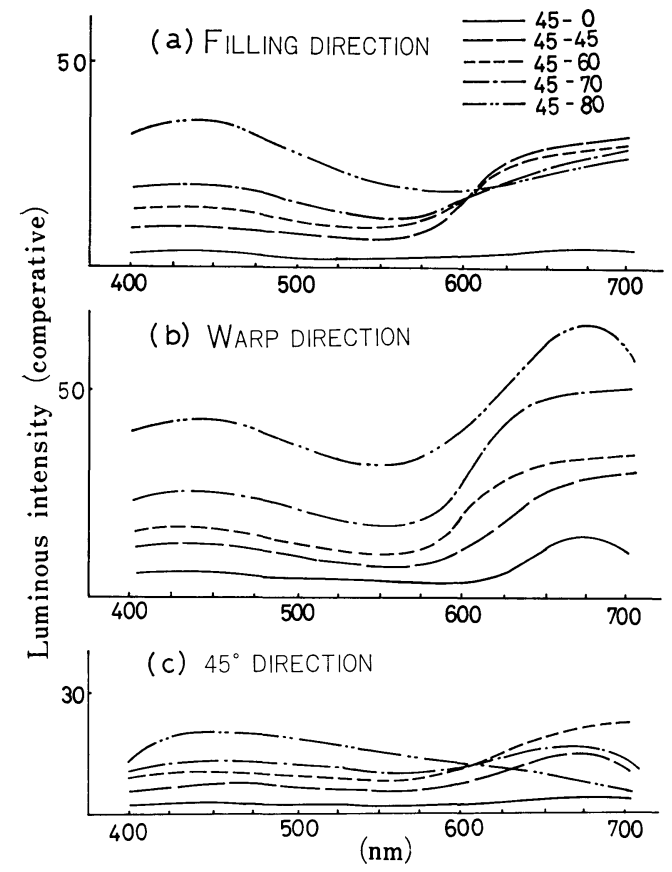

Fig. 17 Monochromatic comparative luminous intensity (for white surface) of Tamamushi fabrics (Sample No. 1)

the spectral reflection curve. The results show that, when the reflected light is measured in the filling direction, the larger the receiving angle the larger the blue color component due to the influence of the warp. When it is measured in the warp direction, as the influence of the filling is large and the reflection from the warp is also bright, so the mixed color becomes reddish.

The goniophotometric curve in the filling direction shows that the blue component is larger than the red component at the receiving angle of about $75^{\circ}$. In the case where it is measured in the warp direction, the larger the receiving angle, the larger the red component.

The results obtained from color measurement (varying the incidence and receiving angles) of samples described in Table 1 are shown in Table 2, and the chromaticity diagrams in Figs. 18 to 20. When measured in the direction of

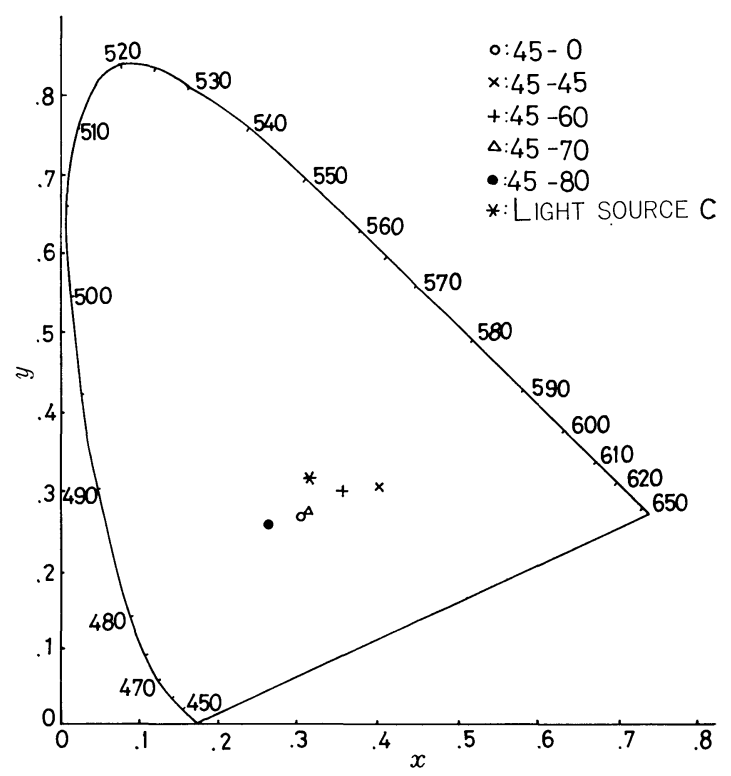

Fig. 18 Color measurements for various directions of Tamamushi fabrics(Sample No. 1. Filling direction)

Table 2 Observed Chromatic Values of Tamamushi Fabrics (Sample No. 3)

\begin{tabular}{c|c|c|c|c|c|c}
\hline $\begin{array}{c}\text { Direction of } \\
\text { measurement }\end{array}$ & $\begin{array}{c}\text { Incidence angle- } \\
\text { Receiving angle }\end{array}$ & $\mathrm{X}$ & $\mathrm{Y}$ & $\mathrm{Z}$ & $\mathrm{x}$ & $\mathrm{y}$ \\
\hline Filling direction & $45-0$ & 15.659 & 14.853 & 6.885 & 0.419 & 0.397 \\
& $45-45$ & 31.789 & 28.468 & 16.553 & 0.414 & 0.371 \\
& $45-60$ & 41.605 & 38.512 & 23.878 & 0.400 & 0.370 \\
& $45-70$ & 52.369 & 51.511 & 33.389 & 0.382 & 0.375 \\
& $45-80$ & 79.721 & 75.721 & 57.018 & 0.375 & 0.356 \\
\hline Warp direction & $45-0$ & 15.927 & 14.313 & 6.888 & 0.429 & 0.385 \\
& $45-45$ & 39.718 & 36.702 & 21.258 & 0.407 & 0.376 \\
& $45-60$ & 51.664 & 48.091 & 28.911 & 0.402 & $0-374$ \\
& $45-70$ & 61.582 & 58.216 & 37.309 & 0.392 & 0371 \\
& $45-80$ & 88.809 & 83.737 & 60.862 & 0.389 & 0.359 \\
\hline \multirow{2}{*}{$45^{\circ}$ direction } & $45-0$ & 14.997 & 13.605 & 5.880 & 0.435 & 0.395 \\
& $45-45$ & 27.598 & 24.883 & 12.941 & 0.422 & 0.380 \\
& $45-60$ & 34.812 & 32.186 & 16.724 & 0.416 & 0.384 \\
& $45-70$ & 40.102 & 42.175 & 23.175 & 0.380 & 0.400 \\
& $45-80$ & 52.682 & 55.072 & 34.397 & 0.371 & 0.387 \\
\hline
\end{tabular}




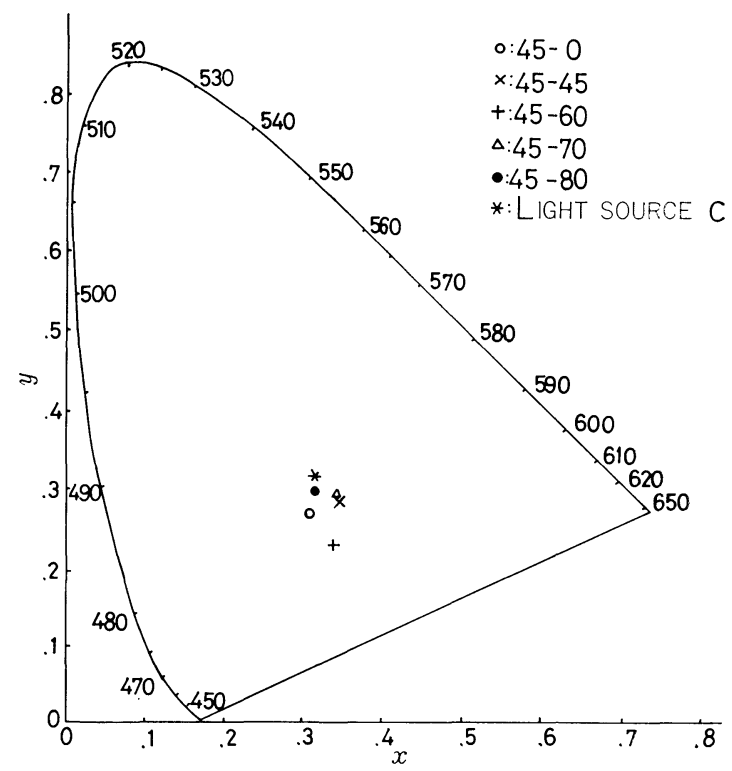

Fig. 19 Color measurements for various directions of Tamamushi fabrics (Sample No. 1. Warp direction)

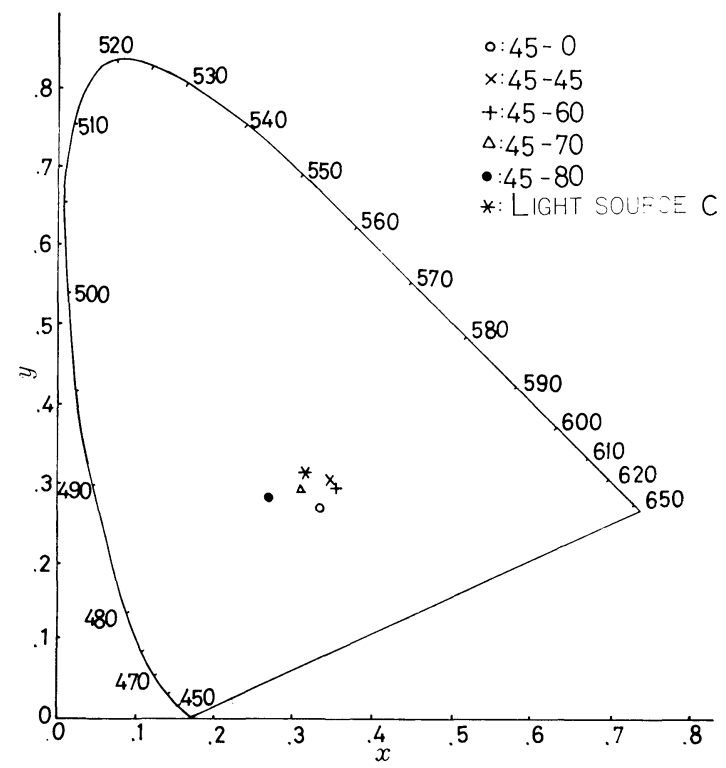

Fig. 20 Color measurements for various directions of Tamamushi fabrics (Sample No. 1 . Left $45^{\circ}$ direction)

the filling, the red component is dominant at the receiving angles of $45^{\circ}$ and $60^{\circ}$, the blue at $80^{\circ}$, and the mixture of both at $0^{\circ}$ and $70^{\circ}$. (Fig. 18) This agrees with the result of the goniophotometric reflection curve in Fig. 12.

When the color is measured in the warp direction, the mixture of both components is observed regardless of the receiving angle (Fig. 19). When the color is measured in $45^{\circ}$ direction against the warp direction, the mixed color of both is shown up except the blue at the receiving angle of $80^{\circ}$. This agrees with the result of the goniophotometric reflection curve measured in the filling direction. That is, the goniophotometrically measured color of Tamamushi fabric can be explained from the ratio of the reflection distribution curve of the warp to that of the filling when the sample is illuminated with the warp and the filling in color.

\section{4-3. Discussion of Tamamushi fabric}

The primary factor for having Tamamushi effect is to give to the same visual cell such stimulus as is from warps and filling having different color. In this case, the iridescent effect is promoted by largely distributed specular reflection which works as a gloss. When two points are brought near and it is difficult to distinguish between the warp color and the filling color, the color change is given rise by the color mixing of warps and fillings.

However, human eyes can find not only the color change due to the color mixing of Tamamushi fabric, but also find 1) the color difference between warp and filling, and 2) the unevenness and waviness of the fabric surface in threedimensional pattern. For example, it is clear that photographs do not always express the real properties of Tamamushi fabric. The optical instruments (goniophotometer, camera, etc.) which have no sense like human being cannot afford the real status of Tamamushi fabric. They only measure the color mixing due to warps and fillings. However, it is not difficult to clarify the optical properties of Tamamushi fabric from reflection distribution curves obtained by the goniophotometer. For example, when the receiving angle is changed at a fixed incident angle, the measured color component varies. The phenomenon such as the view angle is partially varied by the waviness produced on the fabric surface appears continuously or partially on the fabric surface, and this increases the iridescent effect.

When the incident light is parallel with the fiber axis, the specular reflection is large. When it is perpendicular to the fiber axis, the diffuse reflection is small, and the specular reflection in the specular reflection direction of the fabric is decreased.

When the fabric is measured in the filling direction, the warp becomes perpendicular to the photometric plane, and the diffuse reflection is increased, showing the object color.

Reflection from the filling varies with the receiving angle. It shows a light source color, but not the object color in the specular reflection direction.

The variation of the reflection mechanism from the warp or from the filling due to the change of view angles, and the shade made by mutual overlap of warps and fillings makes human being recognize Tamamushi effect.

\section{Conclusions}

The optical properties of Tamamushi fabric were dis- 
cussed from the goniophotometric reflection curves.

The primary factors governing the Tamamushi effect are

1) combination of colors of warps and fillings.

2) threads (filament, cotton, spun yarn, count, material, twist, etc.)

3) fabric structure (plain, twill, sateen, etc.) and its density

4) texture (regularity of surface, unevenness, waviness, pile), directional properties and gloss.

Since Tamamushi fabrics are composed of these elements, it is necessary to discuss them synthetically. Investigation into the color change of Tamamushi fabric should be analyzed from the goniophotometric reflection curves taking these factors into consideration.

\section{Literature cited}

r1] Tanaka, Furusaku; J. Text. Mach. Soc. Japan, 11, T246 (1958)

[2] Sawaji, Motozawa; Pre-print presented at the Textile Machinery Society of Japan, Annual Meeting, p. 155 (May 1967)

[3] Tsuboi, Nihira, Gunji; J. Text. Mach. Soc. Japan, 25, T19 (1972)

[4] Gunji, Nihira, Tsuboi; J. Text. Mach. Soc. Japan, 24, T149 (1971)

[5] Gunji, Nihira, Tsuboi; J. Text. Mach. Soc. Japan, 24, T33 (1971)

[6] Gunji, Nihira, Tsuboi; J. Text. Mach. Soc. Japan, 24, T42 (1971) 